Pacific Journal of Mathematics

ON APPROXIMATION BY DILATION OF DISTRIBUTIONS 


\title{
ON APPROXIMATION BY DILATIONS OF DISTRIBUTIONS
}

\author{
S. R. HARASYMIV
}

Let $E$ be a locally convex space of temperate distributions and suppose that $u \in E$. We attempt to characterize the closed vector subspace of $E$ generated by the set of all distributions having the form $u\left(a_{1} x_{1}+b_{1}, \cdots, a_{n} x_{n}+b_{n}\right)$ where $a_{1}, \cdots, a_{n}$, $b_{1}, \cdots, b_{n}$ are real numbers with $a_{1}, \cdots, a_{n}$ being nonzero. The characterization is effected in the case when the topology on $E$ satisfies certain conditions.

1. Notation. The underlying topological group in all our analysis is the additive group $R^{n}$ with the usual topology. Addition and multiplication in $R^{n}$, are defined component-wise, in the usual manner. We identify the Pontryagin character group of $R^{n}$ with $R^{n}$. Typical elements of $R^{n}$ will be denoted by $x, y, \cdots$, or, when we are thinking of $R^{n}$ as its own character group, by $\chi, \xi, \cdots$. If $\chi \in R^{n}$, then the bounded continuous character of $R^{n}$ corresponding to $\chi$ is defined by

$$
\left(x_{1}, \cdots, x_{n}\right) \rightarrow \exp \left\{-2 \pi i\left(\chi_{1} x_{1}+\cdots+\chi_{n} x_{n}\right)\right\} .
$$

The ordinary Lebesgue measure on $R^{n}$ is denoted by $d x$, or by $d \chi$ if we think of $R^{n}$ as its own character group. With the identification expressed in (1.1), the Fourier Inversion Formula holds without any multiplicative constants.

Throughout, we adopt the usual conventions and notations of the calculus of $n$ variables; see, for example, Hörmander ([5], p. 4). If $x \in R^{n}$, and $k \leqq n$ is a positive integer, we write $x_{k}$ for the $k$-th component of $\alpha$. If $\alpha$ is a multi-index, then the function $j^{\alpha}$ on $R^{n}$ is defined by $j^{\alpha}(x)=x_{1}^{\alpha_{1}} \cdots x_{n}^{\alpha_{n}}$ for all $x \in R^{n}$.

$R^{\sharp}$ will denote the set $R^{n} \backslash\left\{x \in R^{n}: x_{k}=0\right.$ for some $\left.k\right\}$.

Let $W$ be an open set in $R^{n}$. We write $C^{\infty}(W)$ for the set of all complex valued functions which are defined in $W$ and are indefinitely differentiable there. $D(W)$ will denote the set of functions which are indefinitely differentiable and have compact support in $W$. The space of distributions with support in $W$ is denoted by $D^{\prime}(W)$. For an account of these spaces, see Schwartz [6] and [7].

The space of rapidly decreasing indefinitely differentiable functions on $R^{n}$ is designated by $S\left(R^{n}\right)$. The topological dual $S^{\prime}\left(R^{n}\right)$ of $S\left(R^{n}\right)$ is the space of temperate distributions on $R^{n}$. We shall always assume that $S^{\prime}\left(R^{n}\right)$ is equipped with the strong topology $\beta\left(S^{\prime}, S\right)$.

Finally, let $\rho \in D\left(R^{n}\right)$ and suppose that $b \in R^{n}$. Then the function $\rho_{b} \in D\left(R^{n}\right)$ defined by 


$$
\varphi_{b}(x)=\varphi(x+b) \quad \text { for all } x \in R^{n}
$$

is called a translate of $\varphi$. If $a \in R^{*}$, then the function $\varphi^{a} \in D\left(R^{n}\right)$ defined by

$$
\varphi^{a}(x)=\varphi(a x) \quad \text { for all } x \in R^{n}
$$

is called a dilation of $\varphi$. For an arbitrary distribution $u \in D^{\prime}\left(R^{n}\right)$, we define the dilation $u^{a}$ and translate $u_{b}$ of $u$ by making use of the adjoints of the mappings $\varphi \rightarrow \varphi^{a^{-1}}$ and $\varphi \rightarrow \varphi_{b}$; we write $u^{a}(\varphi)=$ $|1 / j(a)| . \quad u\left(\phi^{a^{-1}}\right)$ and $u_{b}(\varphi)=u\left(\varphi_{b}\right)$ for all $\varphi \in D^{\prime}\left(R^{n}\right)$. It is easy to verify that if $u$ is a function, then $u^{a}$ and $u_{b}$ are also functions; and that $u^{a}(x)=u(a x)$ and $u_{b}(x)=u(x+b)$ in this case. [The identification of a function with a distribution is made via the integral, in the usual way.] We notice that for any distribution $u, \operatorname{supp} u^{a}=a^{-1} \cdot \operatorname{supp} u$; and if $u$ is a temperate distribution on $R^{n}$, then $u^{a}$ is a temperate distribution and $\widehat{u^{a}}=|1 / j(a)| \cdot \widehat{u}^{a-1}$. The last equality may be established by an easy computation, or by reference to equation (5.15.14) in Edwards [2].

A vector subspace $F$ of $D^{\prime}\left(R^{n}\right)$ is said to be dilation-invariant [resp. translation-invariant] if $u^{a} \in F\left[u_{b} \in F\right]$ for all $u \in F$ and all $a \in R^{*}$ [all $\left.b \in R^{n}\right]$.

2. $c$-admissible spaces. In this section we shall define the distribution spaces which form the setting for our approximation problems. We shall only consider vector subspaces of $S^{\prime}\left(R^{n}\right)$ which contain $S\left(R^{n}\right)$. To avoid repetition, it will always be understood that whenever we speak of a space of temperate distributions, we mean a vector subspace of $S^{\prime}\left(R^{n}\right)$ which contains $S\left(R^{n}\right)$.

We begin by introducing a modified version of a definition which appears in Yoshinaga and Ogata [8].

Definition 2.1. (cf. Yoshinaga and Ogata [8], p. 17.) Suppose that $E$ is a locally convex space of temperate distributions. We say that $E$ is an admissible space if the following conditions are satisfied.

(i) $S\left(R^{n}\right)$ is dense in $E$.

(ii) The injections $S\left(R^{n}\right) \rightarrow E \rightarrow S^{\prime}\left(R^{n}\right)$ are continuous.

REMARKs. (1) It is easy to see that the topological dual $E^{\prime}$ of an admissible space $E$ is [isomorphic to] a vector subspace of $S^{\prime}\left(R^{n}\right)$ which contains $S\left(R^{n}\right)$; thus we can identify $E^{\prime}$ with a space of temperate distributions. If this identification is made in the obvious way, then

$$
\langle u, \varphi\rangle=u * \varphi(o) \quad \text { for all } u \in E
$$




$$
\langle\varphi, v\rangle=\phi * v(o) \quad \text { for all } v \in E^{\prime}
$$

whenever $\varphi \in S\left(R^{n}\right)$. [Here and elsewhere, $\langle$,$\rangle denotes the bilinear$ form on $E \times E^{\prime}$ arising from the natural pairing of $E$ and $E^{\prime}$.] In view of (2.2), the topology on $E$ is necessarily Hausdorff.

(2) We also remark that if $E$ is admissible according to Definition 2.1, then $E$ is admissible in the sense of Yoshinaga and Ogata [8]. This is easily demonstrated if we notice that the injections $D\left(R^{n}\right) \rightarrow S\left(R^{n}\right)$ and $S^{\prime}\left(R^{n}\right) \rightarrow D^{\prime}\left(R^{n}\right)$ are continuous.

We shall need the following simple result about admissible spaces.

THEOREM 2.2. (a) Let $E$ be a translation-invariant admissible space. If for some $b \in R^{n}$ the mapping $u \rightarrow u_{b}$ of $E$ (with its usual topology) into $E$ (with the weak topology $\sigma\left(E, E^{\prime}\right)$ ) is continuous, then $v_{b} \in E^{\prime}$ for all $v \in E^{\prime}$ and

$$
\left\langle u, v_{b}\right\rangle=\left\langle u_{b}, v\right\rangle \text { for all } u \in E \text { and all } v \in E^{\prime} .
$$

(b) Let $E$ be a dilation-invariant admissible space. If for some $a \in R^{\sharp}$ the mapping $u \rightarrow u^{a}$ of $E$ (with its usual topology) into $E$ (with the weak topology $\sigma\left(E, E^{\prime}\right)$ ) is continuous, then $v^{a^{-1}} \in E^{\prime}$ for all $v \in E^{\prime}$ and

$$
\left\langle u, v^{a^{-1}}\right\rangle=|j(a)| \cdot\left\langle u^{a}, v\right\rangle \text { for all } u \in E \text { and all } v \in E^{\prime} \text {. }
$$

Proof. We shall prove (b); the proof (a) is similar. Suppose that the mapping $u \rightarrow u^{a}$ is continuous for the stated topologies on $E$. Let $v \in E^{\prime}$. Then the mapping $u \rightarrow u^{a} \rightarrow|j(a)| .\left\langle u^{a}, v\right\rangle$ defines a continuous linear functional on $E$, and so is represented by an element $w \in E^{\prime}$. Now, if $\varphi \in S\left(R^{n}\right)$, then in view of (2.2)

$$
\begin{aligned}
\varphi * w(o) & =\langle\varphi, w\rangle \\
& =|j(a)| \cdot\left\langle\varphi^{a}, v\right\rangle \\
& =|j(a)| \cdot \phi^{a} * v(o) \\
& =\varphi * v^{a}(o)
\end{aligned}
$$

the last equality being a consequence of the definition of $v^{a^{-1}}$. Therefore $w=v^{a^{-1}}$ as a temperate distribution, and so $v^{a^{-1}} \in E^{\prime}$. Since $v \in E^{\prime}$ was arbitrary, the proof is complete.

Definition 2.3. Suppose that $E$ is an admissible space. We say that $E$ is $c$-admissible if it satisfies conditions (i)-(iii) below.

(i) $E$ is translation-invariant.

(ii) For each $x \in R^{n}$, the mapping $u \rightarrow u_{x}$ of $E$ (with its usual topology) into $E$ (with the weak topology $\sigma\left(E, E^{\prime}\right)$ ) is continuous. 
(iii) For each $u \in E$ and each $v \in E^{\prime}$, the mapping $x \rightarrow\left\langle u_{x}, v\right\rangle$ defines a continuous function which is a temperate distribution on $R^{n}$.

A $c$-admissible space which satisfies conditions ((iv)-(vi)) below is called a dilation space.

(iv) $E$ is dilation-invariant.

(v) For each $x \in R^{\sharp}$, the mapping $u \rightarrow u^{x}$ of $E$ (with its usual topology) into $E$ (with the weak topology $\sigma\left(E, E^{\prime}\right)$ ) is continuous.

(vi) For each $u \in E$, the mapping $x \rightarrow u^{x}$ of $R^{\sharp}$ into $E$ is continuous for the $\sigma\left(E, E^{\prime}\right)$ topology on $E$.

Dilation spaces form the background against which we shall delineate our approximation problems. This is done in $\S 3$. The remainder of this section is devoted to the derivation of results about $c$-admissible spaces which we shall need in what follows. Throughout the rest of this paper, if $E$ is a $c$-admissible space and $u \in E, v \in E^{\prime}$, then $u \bigcirc v$ will denote the temperate distribution generated by the function $x \rightarrow\left\langle u_{x}, v\right\rangle\left(x \in R^{n}\right)$, as in condition (iii) of Definition 2.3. If we consider $u \circ v$ as a function, then $u \supset v(x)=\left\langle u_{x}, v\right\rangle=\left\langle u, v_{x}\right\rangle$ (by Theorem 2.2 (a)).

Theorem 2.4. (a) If $E$ is a c-admissible space, then $E^{\prime}$ is translation-invariant; moreover, for each $b \in R^{\text {n }}$

$$
u \supset v_{b}=u_{b} \bigcirc v=(u \circ v)_{b} \quad\left(u \in E, v \in E^{\prime}\right) .
$$

(b) If $E$ is a dilation space, then $E^{\prime}$ is dilation-invariant; moreover, for each $a \in R^{\sharp}$

$$
u \circ v^{a^{-1}}=|j(a)| \cdot\left(u^{a} \bigcirc v\right)^{a^{-1}} \quad\left(u \in E, v \in E^{\prime}\right) .
$$

Proof. We shall prove (b); a very similar argument will establish (a).

Assume that $E$ is a dilation space. Then the dilation invariance of $E^{\prime}$ follows at once from Theorem 2.2 (b). Moreover, Theorem 2.2 (b) implies that if $a \in R^{\ddagger}$, then for all $x \in R^{n}$

$$
\begin{aligned}
u \circ v^{a^{1-}}(x) & =\left\langle u_{x}, v^{a^{1-}}\right\rangle \\
& =|j(a)| \cdot\left\langle\left(u_{x}\right)^{a}, v\right\rangle \\
& =|j(a)| \cdot\left\langle\left(u^{a}\right)_{a^{-1} x}, v\right\rangle \\
& =|j(a)| \cdot u^{a} \bigcirc v\left(a^{-1} x\right) \\
& =|j(a)| \cdot\left(u^{a} \bigcirc v\right)^{a^{-1}}(x)
\end{aligned}
$$

which is the result we set out to establish.

THeOREM 2.5. Let $E$ be a barrelled c-admissible space. Then for each $v \in E^{\prime}$, the mapping $u \rightarrow u \circ v$ of $E$ into $S^{\prime}\left(R^{n}\right)$ is continuous. 
Proof. In view of the fact that $E$ is barrelled and $S^{\prime}\left(R^{n}\right)$ is $B_{r}$-complete (Edwards [2], Proposition 8.10.7), the proof of Theorem 2.5 will be complete if we succeed in showing that the graph of the mapping $u \rightarrow u \circ v$ is closed in $E \times S^{\prime}\left(R^{n}\right)$; see Edwards [2], Theorem 8.9.4 and the first remark following it.

Thus, assume that $u \in E, w \in S^{\prime}\left(R^{n}\right)$ and $\left(u_{i}\right)$ is a net in $E$ such that $\lim _{i} u_{i}=u$ in $E$ and $\lim _{i} u_{i} \bigcirc v=w$ in $S^{\prime}\left(R^{n}\right)$. We have to show that $u \circ v=w$.

We first observe that condition (ii) in Definition 2.3 and Theorem 2.2 (a) together imply that the mapping $x \rightarrow v_{x}$ of $R^{n}$ into $E^{\prime}$ is continuous for the weak topology on $E^{\prime}$. Therefore, if $K \subset R^{n}$ is compact, then the set $\left\{v_{x}: x \in K\right\}$ is a weakly compact, and hence weakly bounded, subset of $E^{\prime}$. Since $E$ is barrelled, reference to Edwards [2], Theorem 7.1.1 (b), assures us that the set $\left\{v_{x}: x \in K\right\}$ is equicontinuous. In view of the remark on p. 504 (third paragraph) of Edwards [2], we now infer that

$$
\begin{aligned}
\lim _{i} u_{i} \bigcirc v(x) & =\lim _{i}\left\langle u_{i}, v_{x}\right\rangle \\
& =\left\langle u, v_{x}\right\rangle \\
& =u \bigcirc v(x)
\end{aligned}
$$

uniformly for $x \in K$. The arbitrary nature of the compact set $K \subset R^{n}$ leads us to the conclusion that $\lim _{i} u_{i} \bigcirc v=u \circ v$ uniformly on compact sets.

Now suppose that $\varphi \in D\left(R^{n}\right)$. If we keep in mind the remarks made above, it is easy to see that

$$
\begin{aligned}
u \circ v(\varphi) & =\int_{R^{n}} u \circ v(x) \varphi(-x) d x \\
& =\lim _{i} \int_{R^{n}} u_{i} \bigcirc v(x) \varphi(-x) d x \\
& =\lim _{i} u_{i} \bigcirc v(\varphi) \\
& =w(\varphi)
\end{aligned}
$$

since $\lim _{i} u_{i} \bigcirc v=w$ in $S^{\prime}\left(R^{n}\right)$. Thus $u \circ v=w$ and so the graph of the mapping $u \rightarrow u \circ v$ is closed, as we wished to show.

In $\S 4$, we shall make repeated use of the following result, which is a simple consequence of Theorem 2.5.

Theorem 2.6. Let $E$ be a barrelled c-admissible space. Then $E^{\prime}$ is a module over $S\left(R^{n}\right)$ with respect to convolution. Moreover, if $\varphi \in S\left(R^{n}\right)$, then

$$
(u \circ v) * \varphi=u \circ(v * \varphi) \text { for all } u \in E \text { and all } v \in E^{\prime} .
$$


Proof. Suppose that $v \in E^{\prime}$ and that $\varphi \in S\left(R^{n}\right)$. By Theorem 2.5, the mapping $u \in E \rightarrow u \bigcirc v \in S^{\prime}\left(R^{n}\right) \rightarrow(u \bigcirc v) * \varphi(o)$ defines a continuous linear functional on $E$. This mapping is therefore represented by an element $w \in E$; thus

$$
\langle u, w\rangle=(u \circ v) * \varphi(o) \quad \text { for all } u \in E .
$$

Next we notice that if $\psi \in S\left(R^{n}\right)$, then $\psi \supset v=\psi * v$; this is easily verified if we bear in mind relation (2.2). Therefore, for each $\psi^{\prime} \in S\left(R^{n}\right)$ we have

$$
\begin{aligned}
\psi * w(o) & =\langle\psi, w\rangle \\
& =(\psi \circ v) * \varphi(o) \\
& =\psi * v * \varphi(o) .
\end{aligned}
$$

This entails that $w=v * \varphi$ as a temperate distribution; whence $v * \varphi \in E^{\prime}$. Since $v \in E^{\prime}$ and $\varphi \in S\left(R^{n}\right)$ where arbitrarily chosen, we infer that $E^{\prime}$ is a module over $S\left(R^{n}\right)$.

To complete the proof, notice that (2.3) can now be written as

$$
\langle u, v * \varphi\rangle=(u \bigcirc v) * \varphi(o) \quad \text { for all } u \in E \text {. }
$$

Using (2.4) and Theorem 2.4 (a), we deduce that for $x \in R^{n}$

$$
\begin{aligned}
(u \bigcirc v) * \varphi(x) & =(u \bigcirc v) * \varphi_{x}(o) \\
& =\left(u_{x} \bigcirc v\right) * \varphi(o) \\
& =\left\langle u_{x}, v * \varphi\right\rangle \\
& =u \bigcirc(v * \varphi)(x)
\end{aligned}
$$

which is the required identity.

We end this section with the following theorem, which is of some interest. The convolution to which we refer is defined in Chevalley [1].

THEOREM 2.7. Let $E$ be a barrelled dilation space and suppose that $v \in E^{\prime}$ is such that $u * v$ is defined (in Chevalley's sense) for each $u \in E$ and is a temperate distribution on $R^{n}$. Then $u \bigcirc v=u * v$ for each $u \in E$.

Proof. If $v$ has the stated property, then Theorem 3 (2) and Theorem 2 (2) in Yoshinaga and Ogata [8] together imply that the mapping $u \rightarrow u * v$ of $E$ into $S^{\prime}\left(R^{n}\right)$ is continuous. According to Theorem 2.5 , the mapping $u \rightarrow u \circ v$ of $E$ into $S^{\prime}\left(R^{n}\right)$ is also continuous. Since these two mappings coincide (because of (2.2)) of the dense subset $S\left(R^{n}\right)$ of $E$, they are identical. 
3. Statement of the problem. Let $E$ be a dilation space and suppose that $u \in E$. We denote by $T[u]$ the closed vector subspace of $E$ generated by the set of distributions $\left\{\left(u_{b}\right)^{a}: a \in R^{\sharp}, b \in R^{n}\right\}$. In this paper, we shall be concerned with the task of characterizing $T[u]$ in the case when the topology on $E$ is barrelled.

REMARKs. (1) We make the observation that if $E$ is a semireflexive dilation space, then $E^{\prime}$ is a barrelled dilation space when equipped with its strong topology $\beta\left(E^{\prime}, E\right)$. The fact that $E^{\prime}$ is barrelled is stated in Theorem 8.4.3. in Edwards [2]. If we remember that $S\left(R^{n}\right)$ is reflexive, then the continuity of the injections $S\left(R^{n}\right) \rightarrow$ $E^{\prime} \rightarrow S^{\prime}\left(R^{n}\right)$ is seen to follow from Proposition 8.6.5. in Edwards [2]; this is true whether $E$ is semireflexive or not. The semireflixivity of $E$ together with relation (2.1) ensure that $S\left(R^{n}\right)$ is dense in $E^{\prime}$. Thus $E^{\prime}$ is admissible. The verification of conditions (i)-(vi) in Definition 2.3 is trivial. We refer to Proposition 8.6.5, Edwards [2] and Theorem 2.2 to assure ourselves that (ii) and (v) hold; and the remaining conditions follow from Theorem 2.2.

(2) Suppose that $E$ is a dilation space, $u \in E$; and that $A$ is a dense subset of $R^{\sharp}$ and $B$ is a dense subset of $R^{n}$. Then the closed vector subspace of $E$ generated by the set of distributions

$$
\left\{\left(u_{b}\right)^{a}: a \in A, b \in B\right\}
$$

coincides with $T[u]$. This is a consequence of the Hahn-Banach theorem, in view of the separate continuity of the map $(x, y) \rightarrow\left(u_{y}\right)^{x}$ of $R^{\sharp} \times R^{n}$ into $E$ for the weak topology on $E$.

4. A preliminary lemma. Consider any function $\varphi \in D\left(R^{n}\right)$. Then if $x \in R^{*}$, it is clear that $\varphi^{x^{-1}} \in D\left(R^{*}\right)$. If $u$ is any distribution on $R^{n}$, we define a function $u \nabla \varphi$ on $R^{\ddagger}$ by

$$
u \nabla \varphi(x)=u * \varphi^{x^{-1}}(o) \quad \text { for all } x \in R^{\sharp} .
$$

Lemma 3.1 in Harasymiv [4] states that $u \nabla \varphi \in C^{\infty}\left(R^{\sharp}\right)$; and it is easily shown by induction that for each multi-index $\alpha$

$$
D^{\alpha}(u \nabla \varphi)=\left(1 / j^{\alpha}\right) \sum_{\alpha \leqq \beta} c_{\beta} \cdot u \nabla\left(j^{3} D^{3} \varphi\right)
$$

where the $c_{\beta}$ are constants depending only on $\alpha$ and $\beta$.

We shall prove the following result.

Lemma 4.1. Let $E$ be a barrelled dilation space. Suppose that $u \in E, v \in E^{\prime}, \varphi \in D\left(R^{\sharp}\right), \psi \in D\left(R^{\sharp}\right)$ and $\eta \in S\left(R^{n}\right)$. Then 


$$
\hat{u} \nabla \varphi \cdot \hat{\eta} \cdot \hat{v}=\int_{R^{n}}\{\varphi(y) /|j(y)|\} \mid \widehat{j \mid \psi} * \eta *\left(u^{y-1} \bigcirc v\right)(0) d y .
$$

Proof. Let $u \in E$. Then we can extract a net $\left(k_{k}\right)$ form $S\left(R^{n}\right)$ such that

$$
\lim _{\lambda} k_{\lambda}=u \text { in } E
$$

We first show that if $\varphi \in D\left(R^{\sharp}\right)$ and $\psi \in D\left(R^{\sharp}\right)$, then

$$
\lim \hat{k_{2}} \nabla \varphi \cdot \psi=\widehat{u} \nabla \varphi \cdot \psi \text { in } D\left(R^{n}\right) .
$$

To do this, we argue as follows. Let $\alpha$ be any multi-index. The Leibnitz formula for differentiation shows that $D^{\alpha}\left[\left(\hat{k}_{\lambda}-\hat{u}\right) \nabla \varphi \cdot \psi\right]$ is a sum of terms of the form $a_{\beta} \cdot D^{\beta}\left[\left(\hat{k}_{\lambda}-\hat{u}\right) \nabla \varphi\right] \cdot D^{\alpha-\beta} \psi$ where $a_{\beta}$ are constants depending only on $\alpha$ and $\beta$ and the summation is carried out over all multi-indices $\beta \leqq \alpha$. Therefore the validity of (4.4) will be established if we show that for each multi-index $\alpha$

$$
\lim _{\lambda} D^{\alpha}\left[\left(\hat{k}_{\lambda}-\hat{u}\right) \nabla \varphi\right]=o \text { uniformly on supp } \psi \text {. }
$$

Now, if $\alpha$ is any multi-index, then by relation (4.1)

$$
D^{\alpha}\left[\left(\hat{k}_{\lambda}-\hat{u}\right) \nabla \varphi\right]=\left(1 / j^{\alpha}\right) \cdot \sum_{\beta \leqq \alpha} c_{\beta} \cdot\left(\hat{k}_{\lambda}-\widehat{u}\right) \nabla j^{\beta} D^{\beta} \varphi
$$

where the $c_{\beta}$ are constants depending only on $\alpha$ and $\beta$. Since $j$ is: bounded away from zero on supp $\psi$, it will suffice, in order to establish. (4.5), to show that for each function $\eta \in D\left(R^{\sharp}\right)$.

$$
\lim _{\lambda}\left(\hat{k}_{\lambda}-\hat{u}\right) \nabla \eta=0 \text { uniformly on compact sets . }
$$

Thus, suppose that $\eta \in D\left(R^{\sharp}\right)$ and let $K$ be a compact subset of $R^{\sharp}$. By virtue of the fact that the mapping $x \rightarrow \hat{\eta}^{x}$ of $R^{\sharp}$ into $E^{\prime}$ is continuous for the weak topology on $E^{\prime}$ (by Definition 2.3 and Theorem $2.2(b))$, the set $\left\{\hat{\eta}^{x}: x \in K\right\}$ is weakly compact, and therefore weakly bounded in $E^{\prime}$. Since $E$ is barrelled, this entails that $\left\{\hat{\eta}^{x}: x \in K\right\}$ is: an equicontinuous subset of $E^{\prime}$ (Edwards [2], Theorem 7.1.1 (b)). Secondly, we notice that if $A=\sup \{|1 / j(x)|: x \in K\}$ (observe that $A<\infty)$ then for each $x \in K$ we have

$$
\begin{aligned}
\left|\left(\hat{k}_{\lambda}-\hat{u}\right) \nabla \eta(x)\right| & =\left|\left(\hat{k}_{\lambda}-\hat{u}\right) * \eta^{x^{-1}}(o)\right| \\
& \left.=\mid \hat{k}_{\lambda}-\hat{u}\right)\left(\eta^{x^{-1}}\right) \mid \\
& =\mid\left(k_{\lambda}-u\right) \widehat{\left(\eta^{x-1}\right) \mid} \\
& =|1 / j(x)| \cdot\left|\left(k_{\lambda}-u\right) * \hat{\eta}^{x}(o)\right| \\
& \leqq A \cdot\left|\left\langle k_{\lambda}-u ; \hat{\eta}^{x}\right\rangle\right| .
\end{aligned}
$$


In view of the above facts and relation (4.3), we infer (Edwards [2], p. 504, third paragraph) that

$$
\lim _{\lambda}\left(\hat{k}_{\lambda}-\hat{u}\right) \nabla \eta(x)=0 \text { uniformly for } x \in K \text {. }
$$

Since $\eta$ and $K$ were arbitrarily chosen, we have established (4.6), whence (4.4) follows.

Using (4.4), we see that if $v \in E^{\prime}$, then for each $\psi \in D\left(R^{\sharp}\right)$ and each $\eta \in S\left(R^{n}\right)$ we have

$$
\begin{aligned}
\hat{u} \nabla \varphi \cdot \hat{\eta} \cdot \hat{v}(\psi) & =\hat{\eta} \cdot \hat{v}(\hat{u} \nabla \varphi \cdot \psi) \\
& =\lim _{\lambda} \hat{\eta} \cdot \hat{v}\left(\hat{k_{\lambda}} \nabla \varphi \cdot \psi\right) \\
& =\lim _{\lambda} \eta * v\left(\widehat{\left(\hat{\hat{k}_{\lambda}} \nabla \varphi \cdot \psi\right)} .\right.
\end{aligned}
$$

Now, if $x \in R^{n}$, then

$$
\begin{aligned}
\widehat{\hat{k_{\lambda} \nabla \varphi \cdot \psi}(x)} & =\int_{R^{n}} \exp (-2 \pi i x \chi) \hat{k_{\lambda}} \nabla \varphi(\chi) \psi(\chi) d \chi \\
& =\int_{R^{n}} \exp (-2 \pi i x \chi) \psi(\chi)\left\{\int_{R^{n}} \hat{k}_{\lambda}(\xi) \varphi(-\xi / \chi) d \xi\right\} d \chi \\
& =\int_{R^{n}} \exp (-2 \pi i x \chi) \psi(\chi)\left\{\int_{R^{n}} \hat{k}_{\lambda}(\chi \xi) \varphi(-\xi) \cdot|j(\chi)| d \xi\right\} d \chi \\
& =\int_{R^{n}} \varphi(-\xi)\left\{\int_{R^{n}} \exp (-2 \pi i x \chi) \cdot|j(\chi)| \cdot \psi(\chi) \cdot \hat{k}_{\lambda}^{\xi}(\chi) d \chi\right\} d \xi \\
& =\int_{R^{n}} \varphi(-y)\left\{\mid \widehat{j \mid \psi} * \widehat{k}_{\lambda}^{y}(x)\right\} d y \\
& =\int_{R^{n}} \varphi(-y)\left\{\left|\widehat{j \mid \psi} * \hat{k}_{\lambda}^{-y^{-1}}(x) \cdot 1 /\right| j(y) \mid\right\} d y \\
& =\int_{R^{n}}\{\varphi(y) /|j(y)|\} \mid \widehat{j \mid \psi} * k_{\lambda}^{y^{-1}}(x) d y .
\end{aligned}
$$

Combining relations (4.7) and (4.8), we see that

$$
\begin{aligned}
\hat{u} \nabla \varphi \cdot \hat{\eta} \cdot \hat{v}(\psi) & =\lim _{\lambda} \int_{R^{n}} \eta * v(x)\left[\widehat{\left.\hat{k}_{\lambda} \nabla \varphi \cdot \psi\right]}(-x) d x\right. \\
& =\lim _{\lambda} \int_{R^{n}} \eta * v(x)\left\{\int_{R^{n}}\{\varphi(y) /|j(y)|\} \mid \widehat{j \mid \psi} * k_{\lambda}^{y^{-1}}(-x) d y\right\} d x \\
& =\lim _{\lambda} \int_{R^{n}}\{\varphi(y) /|j(y)|\} \mid \widehat{j \mid \psi} * \eta * k_{\lambda}^{y-1} * v(o) d y \\
& =\lim _{\lambda} \int_{R^{n}} \varphi(y) \cdot k_{\lambda} *(\mid \widehat{j \mid \psi} * \eta * v)^{y}(o) d y
\end{aligned}
$$

the last equality being a consequence of Theorem 2.4 (b) and relation (2.2), since $E^{\prime}$ is a module over $S\left(R^{n}\right)$ (by Theorem 2.6). Now, supp $\varphi$ is compact. Therefore, since for each $w \in E^{\prime}$ the mapping $x \rightarrow w^{x}$ of 
$R^{*}$ into $E^{\prime}$ is continuous for the weak topology on $E^{\prime}$ (by Theorem

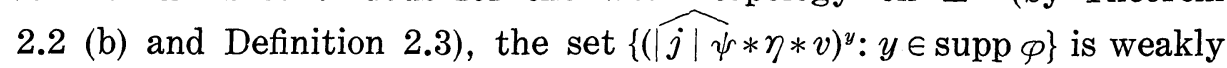
compact and hence weakly bounded in $E^{\prime}$. This set is therefore an equicontinuous subset of $E^{\prime}$ (Edwards [2], Theorem 7.1.1 (b)) and so (by the remark in Edwards [2], p. 504, third paragraph, and relation (2.1))

$$
\begin{aligned}
\lim _{\lambda} k_{\lambda} *(\mid \widehat{j \mid \psi} * \eta * v)^{y}(o) & =\lim _{\lambda}\left\langle k_{\lambda},(\widehat{j \mid \psi} * \eta * v)^{y}\right\rangle \\
& =\left\langle u,(\mid \widehat{j \mid \psi} * \eta * v)^{y}\right\rangle \\
& =|1 / j(y)| \cdot\left\langle u^{y-1}, \mid \widehat{j \mid \psi} * \eta * v\right\rangle
\end{aligned}
$$

uniformly for $y \in \operatorname{supp} \varphi$. Using this in (4.9), we deduce that

$$
\begin{aligned}
\hat{u} \nabla \varphi \cdot \hat{\eta} \cdot \hat{v}(\psi) & =\int_{R^{n}}\left\{\varphi(y) /|j(y)|\left\langle\left\langle u^{y-1}, \widehat{j \mid \psi} * \eta * v\right\rangle d y\right.\right. \\
& =\int_{R^{n}}\{\varphi(y) /|j(y)|\} u^{y^{-1}} \bigcirc(\mid \widehat{j \mid \psi} * \eta * v)(o) d y \\
& =\int_{R^{n}}\{\varphi(y) /|j(y)|\} \cdot \mid \widehat{j \mid \psi} * \eta *\left(u^{y^{-1}} \bigcirc v\right)(o) d y
\end{aligned}
$$

the last equality following from Theorem 2.6. This completes the proof of Lemma 4.1.

Corollary. Let $E$ be a barrelled dilation space. Suppose that $u \in E$ and $v \in E^{\prime}$ are such that $u^{a} \bigcirc v=0$ for all $a \in R^{\sharp}$. Then for each $\varphi \in D\left(R^{\sharp}\right)$ and each $\eta \in S\left(R^{n}\right), \widehat{u} \nabla \varphi \cdot \hat{\eta} \cdot \hat{v}=0$ on $R^{\sharp}$.

5. The main result. The result in the preceding section enables us to prove the following theorem.

THEOREM 5.1. Let $E$ be a barrelled dilation space with the following property:

(i) If $v \in E^{\prime}$ and $\operatorname{supp} \hat{v} \cap R^{\sharp}=\varnothing$, then $v=0$.

Then for each $u \in E$ such that

(ii) $\operatorname{supp} \hat{u} \cap R^{\sharp} \neq \varnothing$

we have $T[u]=E$.

Proof. We first notice that if $u \in E$ is such that (ii) holds, then since $\operatorname{supp} \widehat{u^{a}}=a$. $\operatorname{supp} \hat{u}$, we have

$$
R^{\sharp} \subset \bigcup\left\{\operatorname{supp} \widehat{u^{a}}: a \in R^{\sharp}\right\} .
$$

Now let $v \in E^{\prime}$ be such that

$$
\left\langle\left(u_{b}\right)^{a}, v\right\rangle=0 \text { for all } a \in R^{\sharp} \text { and all } b \in R^{n} .
$$


We shall show that (5.2) implies that supp $\hat{v} \cap R^{*}=\varnothing$. Condition (ii) in the statement of Theorem 5.1 will then entail that $v=0$; and an application of the Hahn-Banach theorem will complete the proof.

Thus, suppose that $\chi \in R^{\ddagger}$. By virtue of (5.1), there exists $c \in R^{\sharp}$ such that $\chi \in \operatorname{supp} \hat{u}^{c}$. Choose a relatively compact neighbourhood $W$ of $\chi$ such that $\bar{W} \subset R^{*}$, and a function $\eta \in S\left(R^{n}\right)$ such that $\hat{\eta}=1$ on $\bar{W}$. Next we notice that since $\chi \in \operatorname{supp} \widehat{u}^{c}$, there exists $\varphi \in D(W)$ such that $\widehat{u}^{c}(\varphi) \neq 0$; that is $\widehat{u}^{c} \nabla \varphi^{x^{-1}}(\chi) \neq 0$. Now $\hat{u}^{c} \nabla \varphi$ is indefinitely differentiable on $R^{\sharp}$; whence (because of (5.3)) we infer that $\hat{u}^{c} \nabla \varphi$ is bounded away from zero on some neighborhood $V \subset W$ of $\chi$. Therefore there exists a function $f \in C^{\infty}\left(R^{z}\right)$ such that $f \cdot \hat{u}^{c} \nabla \varphi=1$ on $V$.

We can now show that supp $\hat{v} \cap R^{*}=\varnothing$ if $v \in E^{\prime}$ satisfies (5.2). It is immediate that if (5.2) holds then $u^{a} \bigcirc v=0$ for all $a \in R^{\sharp}$. In view of the choice of the functions $\eta$ and $f$ above, we have for each $\psi \in D(V)$

$$
\begin{aligned}
\widehat{v}\left(\psi^{\prime}\right) & =\hat{v}\left(f \cdot \widehat{u^{c}} \nabla \phi^{x^{-1}} \cdot \hat{\eta} \psi\right) \\
& =\widehat{u^{c}} \nabla \varphi^{x^{-1}} \cdot \hat{\eta} \cdot \hat{v}(f \psi) \\
& =0
\end{aligned}
$$

since $\widehat{u^{c}} \nabla \varphi^{x^{-1}} \cdot \hat{\eta} \cdot \hat{v}=0$ on $R^{\sharp}$, by the corollary to Lemma 4.1. Hence we infer that $\hat{v}=0$ on $V$, and so $\chi \notin \operatorname{supp} \hat{v}$. Since $\chi \in R^{\ddagger}$ was arbitrarily chosen, it follows that $\operatorname{supp} \hat{v} \cap R^{*}=\varnothing$.

Theorem 5.1 has the following corollary.

THEOREM 5.2. Let E be a barrelled dilation space with the following properties:

(i) $u * \varphi \in C_{0}\left(R^{n}\right)$ for all $u \in E$ and all $\varphi \in D\left(R^{n}\right)$.

(ii) $v * \varphi \in C_{0}\left(R^{n}\right)$ for all $v \in E^{\prime}$ and all $\varphi \in D\left(R^{n}\right)$. Then for each $u \in E, u \neq 0$, it is true that $T[u]=E$.

Proof. Suppose that $v \in E^{\prime}$ and that $\operatorname{supp} \hat{v} \cap R^{\sharp}=\varnothing$. Then $\operatorname{supp} \hat{\varphi} \cdot \hat{v} \cap R^{\#}=\varnothing$ for each $\varphi \in D\left(R^{n}\right)$. In view of condition (ii) above we may argue as in the proof of Theorem 4.2 in Edwards [3] and deduce that $\varphi * v=0$ for all $\varphi \in D\left(R^{n}\right)$. It now follows that $v=0$ and so condition (i) of Theorem 5.1 is satisfied. Similarly, condition (i) of Theorem 5.2 entails that supp $\hat{u} \cap R^{\sharp} \neq \varnothing$ for each nonzero $u \in E$. Now apply Theorem 5.1.

The spaces $C_{0}\left(R^{n}\right)$ (with the uniform norm topology), $L^{b}\left(R^{n}\right)$, $1 \leqq p<\infty$ (with the norm topology), $D_{L p}$ and $D_{L p}^{\prime}, 1 \leqq p<\infty$ (see Schwartz [7]) are some obvious examples of barrelled dilation spaces which satisfy conditions (i) and (ii) in Theorem 5.2. Theorem 1 in Harasymiv [4] is a particular case of Theorem 5.2 above. 


\section{BIBLIOGRAPHY}

1. C. Chevalley, Theory of distributions, lectures at Columbia University, 1950-51.

2. R. E. Edwards, Functional analysis: Theory and applications, Holt, Rinehart and Winston, New York, 1965.

3. - Spans of translates in $L^{p}(G)$, J. Austral. Math. Soc. 5 (1965), 216-233.

4. S. R. Harasymiv, A note on dilations in $L^{p}$, (to appear, Pacific J. Math.)

5. L. Hörmander, Linear partial differential operators, Springer-Verlag, Heidelberg, 1963.

6. L. Schwartz, Théorie des Distributions, tome I. Hermann, Paris, 1950.

7. - Théorie des Distributions, tome II, Hermann, Paris, 1951.

8. K. Yoshinaga, and H. Ogata, On convolutions, J. Sci. Hiroshima Univ. (A) 22 (1958), 15-24.

Received August 9, 1967.

UNIVERSITY OF SYDNEY 


\section{PACIFIC JOURNAL OF MATHEMATICS}

\section{EDITORS}

H. ROYDEN

Stanford University

Stanford, California

\section{R. $R$ PHELPS}

University of Washington

Seattle, Washington 98105

\section{J. DugunduI}

Department of Mathematics

University of Southern California

Los Angeles, California 90007

RICHARD ARENS

University of California

Los Angeles, California 90024

\section{ASSOCIATE EDITORS}

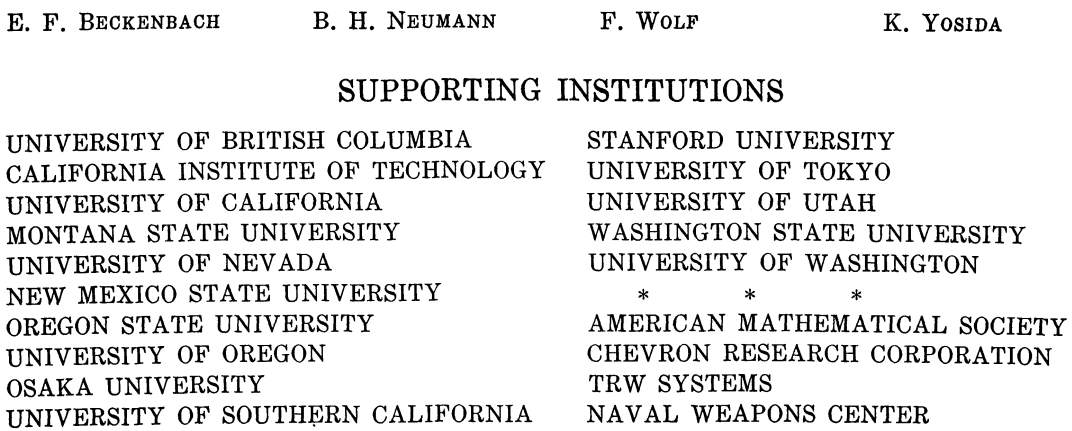

The Supporting Institutions listed above contribute to the cost of publication of this Journal, but they are not owners or publishers and have no responsibility for its content or policies.

Mathematical papers intended for publication in the Pacific Journal of Mathematics should be in typed form or offset-reproduced, double spaced with large margins. Underline Greek letters in red, German in green, and script in blue. The first paragraph or two must be capable of being used separately as a synopsis of the entire paper. It should not contain references to the bibliography. Manuscripts, in duplicate if possible, may be sent to any one of the four editors. Please classify according to the scheme of Math. Rev. 36, 1539-1546. All other communications to the editors should be addressed to the managing editor, Richard Arens, University of California, Los Angeles, California, 90024.

50 reprints are provided free for each article; additional copies may be obtained at cost in multiples of 50 .

The Pacific Journal of Mathematics is published monthly. Effective with Volume 16 the price per volume (3 numbers) is $\$ 8.00$; single issues, $\$ 3.00$. Special price for current issues to individual faculty members of supporting institutions and to individual members of the American Mathematical Society: $\$ 4.00$ per volume; single issues $\$ 1.50$. Back numbers are available.

Subscriptions, orders for back numbers, and changes of address should be sent to Pacific Journal of Mathematics, 103 Highland Boulevard, Berkeley, California, 94708.

PUBLISHED BY PACIFIC JOURNAL OF MATHEMATICS, A NON-PROFIT CORPORATION

Printed at Kokusai Bunken Insatsusha (International Academic Printing Co., Ltd.), 7-17, Fujimi 2-chome, Chiyoda-ku, Tokyo, Japan. 


\section{Pacific Journal of Mathematics}

Vol. 28, No. $2 \quad$ April, 1969

Richard Arens and Donald George Babbitt, The geometry of relativistic

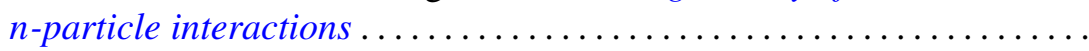

Kirby Alan Baker, Hypotopological spaces and their embeddings in lattices

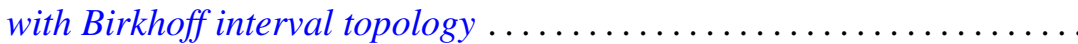

J. Lennart (John) Berggren, Finite groups in which every element is conjugate to its inverse ........................... 289

Beverly L. Brechner, Homeomorphism groups of dendrons . . . . . . . . . . . 295

Robert Ray Colby and Edgar Andrews Rutter, QF - 3 rings with zero singular ideal ................................. 303

Stephen Daniel Comer, Classes without the amalgamation property....... 309

Stephen D. Fisher, Bounded approximation by rational functions ......... 319

Robert Gaines, Continuous dependence for two-point boundary value problems..................................... 327

Bernard Russel Gelbaum, Banach algebra bundles ............... 337

Moses Glasner and Richard Emanuel Katz, Function-theoretic degeneracy criteria for Riemannian manifolds ...................... 351

Fletcher Gross, Fixed-point-free operator groups of order $8 \ldots \ldots \ldots \ldots 357$

Sav Roman Harasymiv, On approximation by dilations of distributions . . . . 363

Cheong Seng Hoo, Nilpotency class of a map and Stasheff's criterion ... . . 375

Richard Emanuel Katz, A note on extremal length and modutus.......... 381

H. L. Krall and I. M. Sheffer, Difference equations for some orthogonal polynomials ................................

Yu-Lee Lee, On the construction of lower radical properties ........... 393

Robert Phillips, Liouville's theorem........................... 397

Yum-Tong Siu, Analytic sheaf cohomology groups of dimension $n$ of

n-dimensional noncompact complex manifolds ..... . .

Michael Samuel Skaff, Vector valued Orlicz spaces. II...

James DeWitt Stein, Homomorphisms of $B^{*}$-algebras .... . .

Mark Lawrence Teply, Torsionfree injective modules .... . . .

Richard R. Tucker, The $\delta^{2}$-process and related topics. II .

David William Walkup and Roger Jean-Baptiste Robert Wets, Lifting

projections of convex polyhedra...

Thomas Paul Whaley, Large sublattices of a lattice. 